\title{
HIV-1 diversity in infected individuals in Suzhou and Suqian, China
}

\author{
Chenhao Qin ${ }^{1 \dagger}$, Ping Zhang ${ }^{1 \dagger}$, Weiguang Zhu ${ }^{2}$, Fangyuan $\mathrm{Hao}^{3}$, Aiping $\mathrm{Gu}^{1}$, Ping Fen ${ }^{1}$, Xueming Zhu ${ }^{1}$ \\ and Hong Du ${ }^{1 *}$
}

\begin{abstract}
Jiangsu is one province with severe HIV-1 epidemic in China. However, the molecular epidemiological characterizations of HIV-1 in many cities of Jiangsu remain unclear. A molecular epidemiological investigation was performed based on 38 HIV-positive samples collected from Suzhou and Suqian during 2011-2013. Five HIV-1 genomic fragments, p17, pol, vif-vpr, vpr-env, and C2V3 were amplified and sequenced from these samples. HIV-1 group M subtype of each sample was determined by phylogenetic analyses with the standard reference sequences. Among these infected individuals, $81.6 \%$ (31/38) self-reported to be infected via sexual contacts, including $50.0 \%$ (19/38) via heterosexual contact and $31.6 \%$ (12/38) via homosexual contact. Among 34 samples with available pol or vif-env sequence, 19 (55.9\%) CRF01_AE, 7 (20.6\%) CRF07_BC, 3 (8.8\%) CRF08_BC, and 5 (14.7\%) inter-subtype recombinants were identified. No pure $B, B^{\prime}$ and $C$ subtypes were found in this cohort. The five recombinants contain one $B / C$, three CRF01/B and one CRF01/B/C recombinants. These results suggest that CRF01_AE was the most predominant HIV-1 group M subtype and CRF01_AE-involved recombinants were the major recombinant forms. Comparison showed that there was no obvious difference in HIV-1 group M subtype distribution between Jiangsu (including Suzhou and Suqian) and the surrounding provinces (e.g., Shanghai, Anhui, and Shandong). CRF01_AE and CRF07_BC were the top two predominant HIV-1 genotypes in Jiangsu, and less and/or no pure subtype B and C was currently circulating here. We predicted that more CRF01/CRF07 recombinants, but fewer B/C recombinants will be generated in Jiangsu in future.
\end{abstract}

Keywords: HIV-1, Epidemiology, Recombination forms, Subtype distribution, CRF01_AE

\section{Background}

HIV/AIDS continues to be one of major public health issues in China. By the end of 2011, there were approximately 780,000 (620,000-940,000) persons living with HIV/AIDS (PLHIV) in China (Ministry of Health of the People's Republic of China, Joint United Nations Programme on HIV/AIDS, World Health Organization 2012). The prevalence rates was $0.058 \%$ (0.046-0.070 \%) nationally. According to the case reporting system, all 31 provinces (including autonomous regions and municipalities) have reported HIV/AIDS cases. The numbers of

\footnotetext{
*Correspondence: hong_du@126.com

${ }^{\dagger}$ Chenhao Qin and Ping Zhang contributed equally to this work

${ }^{1}$ Department of Clinical Laboratory, The Second Affiliated Hospital of Soochow University, 1055 Sanxiang Road, Suzhou 215004, Jiangsu, China

Full list of author information is available at the end of the article
}

infected cases were very different between provinces. The worst-hit provinces by HIV/AIDS in China are Yunnan, Xinjiang, Guangxi and Sichuan, accounting for $75.8 \%$ of the national total (Ministry of Health of the People's Republic of China, Joint United Nations Programme on HIV/AIDS, World Health Organization 2012; Li et al. 2013). Jiangsu, a province in the East China, which neighbors with Shanghai, Zhejiang, Anhui, Henan and Shandong, is one of regions with severe HIV-1 epidemic (Ministry of Health of the People's Republic of China, Joint United Nations Programme on HIV/AIDS, World Health Organization 2012; Zhang et al. 2013; Guo et al. 2009a).

As one of the richest regions of China, Jiangsu is a typical labor force-import province and attracts a large number of migrant populations to work and live (Meng et al. 2011). The data in 2011 showed an obvious increase 
(17.3 \%) in new HIV infections in Jiangsu compared to the data in 2010 (Control JPCfDPA 2012). About $72.0 \%$ of PLHIV in Jiangsu were reported in five cities, Nanjing, Suzhou, Wuxi, Changzhou and Xuzhou (Control JPCfDPA 2012). Molecular epidemiological investigations based on $p 17$ and $C 2 \mathrm{~V} 3$ regions of HIV genome showed that majority of HIV-1 infection in Jiangsu were caused by HIV-1 group M CRF01_AE, B and C subtypes, and few were associated with HIV-1 recombinants (Control JPCfDPA 2012). The co-circulation of multiple HIV-1 group $M$ subtypes in Jiangsu will provide more chances to generate new inter-subtype recombinants. As HIV-1 recombination often occurs in the pol and vif-env regions (Pang et al. 2012), some recombinants in Jiangsu might not be found by the analyses of $p 17$ and $C 2 V 3$ sequences. Recently, some new HIV-1 recombinants were reported in Jiangsu and several surrounding provinces, such as Anhui, Shanghai, Shandong (Wu et al. 2013; Zhong et al. 2007; Zhang et al. 2010; Guo et al. 2009b, 2014). Therefore, the genetic diversity of HIV-1 in Jiangsu might be underestimated, and some new intersubtype recombinants might exist but not be identified. Furthermore, the molecular epidemiological characterizations of HIV-1 in some cities of Jiangsu are still unavailable now.
In this study, we used multiple genomic fragments ( $p 17$, pol, vif-env and C2V3) of HIV-1 to determine the subtype characterization of HIV-1 circulating in two cities (Suzhou and Suqian) of Jiangsu (Fig. 1a). We found that multiple HIV-1 subtypes, including CRF07_BC, CRF08_ BC, CRF01_AE and some inter-subtype recombinants were circulating in the two sampled cities. Our findings provided new molecular epidemiological information for the prevention and control of HIV-1 in Jiangsu and even East China.

\section{Methods}

\section{Samples, HIV-1 RNA extraction and gene fragments amplification}

A total of 38 confirmed HIV sero-positive samples from out-patients and hospitalized patients which were collected from the Second Affiliated Hospital of Soochow University and Suqian CDC. All collected plasma were prepared and stored in a $-80{ }^{\circ} \mathrm{C}$ freezer until use according to standard procedures. Viral RNAs were extracted from $200 \mu \mathrm{l}$ of plasma with the MiniBEST Viral RNA/ DNA Extraction Kit Ver4.0 (TaKaRa Biotechnology Co. Ltd., Dalian, China) and then subjected to the amplification of five HIV-1 genomic fragments ( $p 17, p o l, v i f-v p r$, vpr-env, C2V3). Their locations in HXB2 are 683-1255, 2147-3462, 5084-5805, 5653-6454, and 6817-7381 nt,

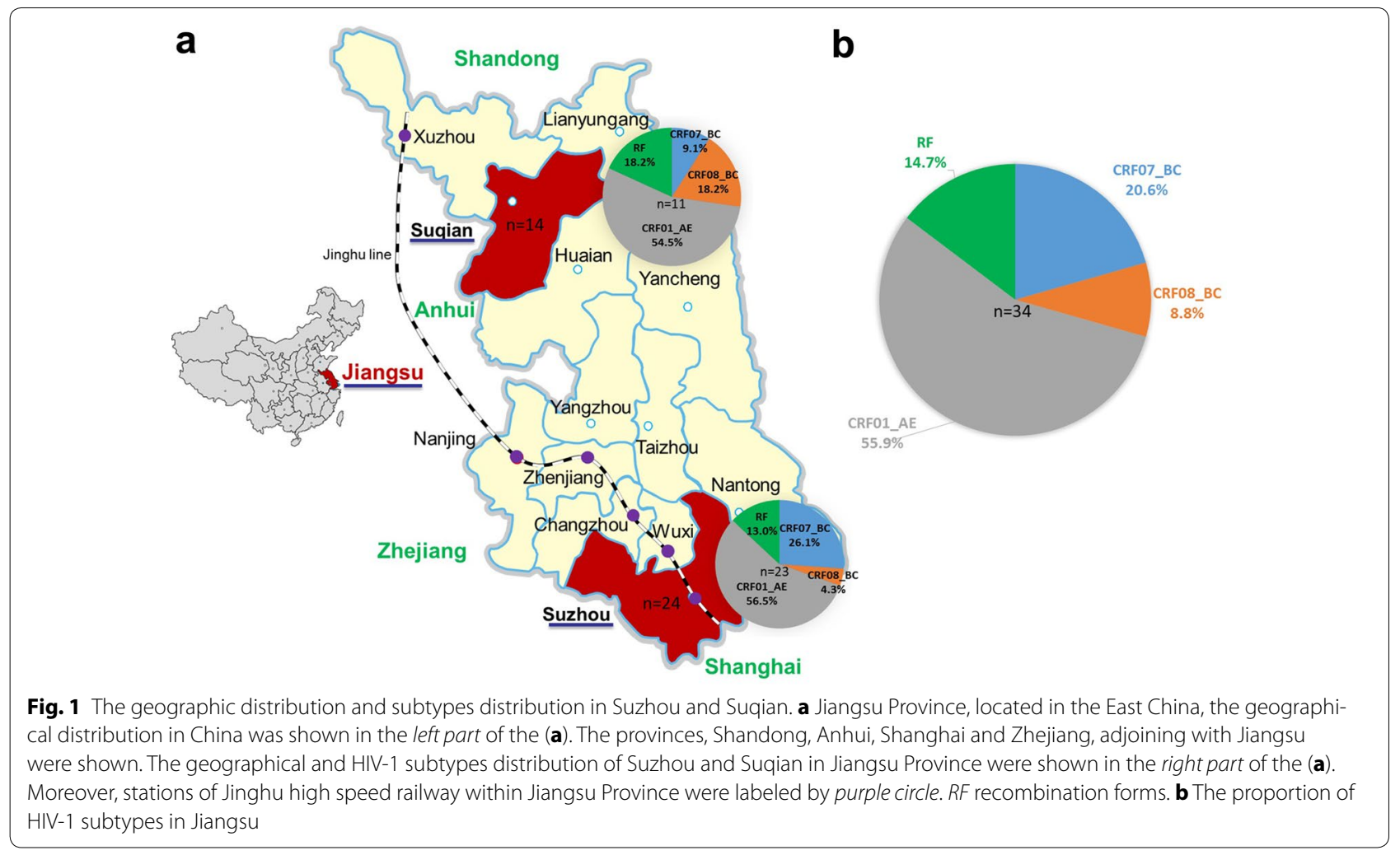


for $p 17, p o l, v i f-v p r, v p r-e n v, C 2 V 3$, respectively. Reverse transcription PCR reactions were performed using One-step RNA PCR kit (TaKaRa Biotechnology, Dalian, China). The PCR products were subjected to second PCR (nested PCR) using ExTaq (TaKaRa Biotechnology, Dalian, China). The primer pairs used in this study and the amplification fragments information were consistent with a previous study (Pang et al. 2012). The condition and the procedure of reverse transcription reaction and PCR reactions were performed according to the operation manual. All amplified products were sent to Shanghai Invitrogen Biotechnology Co., LTD. for sequencing.

\section{HIV genotyping and phylogenetic analyses}

HIV reference sequences were downloaded from Los Alamos HIV Sequence Database (www.hiv.lanl.gov). The obtained sequences were aligned together with reference sequences using the Clustal W program implemented in MEGA 5.2 (Tamura et al. 2011), and then manually edited. The phylogenetic trees were constructed using NJ (neighbor-joining) method with a bootstrap evaluation of 1000 replications with MEGA5.2. To determine the potential recombination of HIV-1, the bootscan analyses were performed by SimPlot 3.5.1 software (Lole et al. 1999). The parameters of SimPlot bootscan analysis were as follows: window size, $200 \mathrm{bps}$; step size, $20 \mathrm{bps}$; tree algorithm, neighbor; distance model, Kimura; bootstrap replicate, 100; reference type, $50 \%$ consensus.

\section{GenBank accession numbers}

The obtained nucleotide sequences of this study have been submitted to GenBank and are available under the accession numbers of KM054876-KM054975.

\section{Results}

\section{Social-demographic characterization of HIV-1 infected} individuals in two cities (Suzhou and Suqian) of Jiangsu

A total of 38 HIV-infected individuals, including 24 (63.2 \%) from Suzhou and 14 (36.8\%) from Suqian, were covered in this study. The social-demographic information, including gender, marital status, educational level, age, ethnic, occupation, risk behaviors, is listed in Table 1. Majority of these individuals are male (84.2\%) and ethnic Han (94.7 \%). Their marriage status showed that $57.9 \%(22 / 38)$ were married, and the others were unmarried, divorced or without this information. More than half (55.3\%) of them were 31-40 years old and $44.7 \%$ were unemployed. Sexual contacts, including heterosexual $(50.0 \%)$ and homosexual (31.6 \%) contacts, were the major risk behavior for HIV-1 infection, accounting for $81.6 \%$ of total. Injecting drug use (IDU) was the second most common factor associated with HIV infection, obviously different from the observations
Table 1 Social-demographic characteristics of HIV-1 infected Suzhou and Suqian

\begin{tabular}{|c|c|c|c|}
\hline Variable & Suzhou & Suqian & Count \\
\hline Location & 24 & 14 & 38 \\
\hline \multicolumn{4}{|l|}{ Gender } \\
\hline Male & 22 & 10 & $32(84.2)$ \\
\hline Female & 2 & 4 & $6(15.8)$ \\
\hline \multicolumn{4}{|l|}{ Marital status } \\
\hline Unmarried & 7 & 3 & 10 (26.3\%) \\
\hline Married & 14 & 8 & $22(57.9 \%)$ \\
\hline Divorced & 3 & 2 & $5(13.2 \%)$ \\
\hline Not clear & 0 & 1 & $1(2.6 \%)$ \\
\hline \multicolumn{4}{|l|}{ Education level } \\
\hline$\leq$ Primary school & 1 & 4 & $5(13.2 \%)$ \\
\hline Middle school & 8 & 6 & 14 (36.8 \%) \\
\hline$\geq$ High school & 15 & 4 & 19 (50.0 \%) \\
\hline \multicolumn{4}{|l|}{ Age (years old) } \\
\hline $15-20$ & 1 & 0 & $1(2.63 \%)$ \\
\hline $21-30$ & 9 & 0 & $9(23.68 \%)$ \\
\hline $31-40$ & 10 & 11 & $21(55.26 \%)$ \\
\hline $41-50$ & 3 & 3 & $6(15.79 \%)$ \\
\hline $51-60$ & 1 & 0 & 1 (2.63 \%) \\
\hline \multicolumn{4}{|l|}{ Nationality } \\
\hline Han & 23 & 13 & $36(94.74 \%)$ \\
\hline Dai & 0 & 1 & $1(2.6 \%)$ \\
\hline Hani & 1 & 0 & $1(2.6 \%)$ \\
\hline \multicolumn{4}{|l|}{ Occupation } \\
\hline Farmer & 0 & 3 & $3(7.9 \%)$ \\
\hline Worker & 5 & 3 & $8(21.1 \%)$ \\
\hline Businessman & 3 & 1 & $4(10.5 \%)$ \\
\hline Unemployment & 13 & 4 & $17(44.7 \%)$ \\
\hline Servicer & 3 & 3 & $6(15.8 \%)$ \\
\hline \multicolumn{4}{|l|}{ Mode of contracted HIV } \\
\hline Homosexual contact & 6 & 6 & $12(31.6 \%)$ \\
\hline Heterosexual contact & 14 & 5 & $19(50.0 \%)$ \\
\hline Injection drug use & 3 & 1 & $4(10.5 \%)$ \\
\hline Uncertain & 1 & 2 & $3(7.9 \%)$ \\
\hline \multicolumn{4}{|l|}{ Dual sex partner } \\
\hline Yes & 0 & 3 & $3(7.89 \%)$ \\
\hline No & 24 & 11 & $35(92.11 \%)$ \\
\hline
\end{tabular}

in southwestern (e.g., Yunnan and Guangxi) and northwestern (e.g., Xinjiang) China (Zhang et al. 2002; Liu et al. 2006, 2008; Sun et al. 2011; Yan et al. 2006).

There are several light differences in social-demographic characterizations between Suzhou and Suqian although the little differences between two cities might be a result of a bias due to small sample size. Most HIVinfected individuals (62.5\%) in Suzhou had completed their high school education, higher than those $(28.6 \%)$ 
in Suqian. Heterosexual contact (58.3\%) was the likely predominant risk factor for HIV infection in Suzhou, while homosexual contact (46.2 \%) was the major factor for HIV infection in Suqian. In addition, three individuals $(21.4 \%)$ in Suquan self-reported to have multiple sex partners, while no one was in Suzhou.

\section{The amplification of HIV genomic fragments}

From 38 HIV-positive samples, 34 (89.5 \%) p17, 32 (84.2\%) pol, 35 (92.1\%) vif-vpr, 32 (84.2\%) vpr-env and $34(89.5 \%) C 2 V 3$ fragments were successfully amplified and sequenced (Table 2). The failure in the amplification of viral genome fragments may be due to primer specificity and low viral load in some specimens. As described in the previous study (Pang et al. 2012), the vif-vpr (5084$5805 \mathrm{nt}$ in HXB2) fragment overlapped with the vprenv (5653-6454 nt in HXB2) fragment, we merged both two fragments into a vif-env sequence if both sequences available for the same sample. Finally, 27 merged vif-env sequences were obtained (Table 2).

\section{Subtyping of HIV-1 based on four HIV-1 genomic fragments}

To investigate the subtype characterization of HIV-1 in Suzhou and Suqian, four phylogenetic trees were constructed based on $p 17$, pol, vif-env and $\mathrm{C} 2 \mathrm{~V} 3$ fragments. Among 34 p17 sequences, 1 (2.9\%), 13 (38.2 \%) and 20 (58.8 \%) were identified to be HIV-1 group M subtype B, C and CRF01_AE, respectively (Fig. 2). In C2V3 tree, except one sequence (11jssqIDU011) that clusters outside the subtype B clade, 11 (32.4\%) and 22 (64.7\%) were clearly identified as HIV-1 group $M$ subtype $C$ and CRF01_AE, respectively (Fig. 3). The strain outside the clades of known subtypes might represent a new inter-subtype recombinant. To determine whether 11 jssqIDU011 is a recombinant, the bootscan analysis was performed using Simplot software. The result revealed that 11jssqIDU011 was a B/C recombinant (Fig. 2).

HIV-1 pol and vif-env fragments are the crucial regions for the confirmation of HIV-1 group $M$ genotype, especially for the finding of new recombinant. So, the pol and vif-env sequences from Suzhou and Suqian were also analyzed. The phylogenetic tree of $\mathrm{pol}$ showed that one sequence (11jssqMSM014) was unable to cluster within the clades of known subtypes (Fig. 3), implying that it might be a recombinant. The bootscan analysis confirmed it being CRF01_AE/B recombinant. To avoid the omission of some recombinants, all other sequences were also subjected to the bootscan analysis in despite that they well clustered within the clades of known subtypes/CRFs in the phylogenetic tree. The bootscan analysis showed that another sequence clustering within the subtype B clade was a $\mathrm{B} / \mathrm{C}$ recombinant (Fig. 3). As a result, 1 (3.1\%) subtype B, 7 (21.9\%) CRF07_BC, 3 (9.4\%) CRF08_BC, 19 (59.4 \%) CRF01_AE and 2 (6.3 \%) inter-subtype recombinants were identified based on 32 pol sequences (Table 2). In the tree of vif-env, all sequences clustered within the known subtype/CRF clades. Interestingly, we found that a sequence from Suzhou clustered with a CRF51_01B sequence. CRF51_01B was firstly identified in Singapore, and mainly prevalent here (Ng et al. 2012). To confirm whether it was CRF51_01B, further bootscan analysis was performed. The result showed that this sequence had different recombination breakpoint with CRF51_01B (Fig. 3), indicating that it was a new CRF01/B recombinant, rather than CRF51_01B. As a result, 1 (3.7 \%) B, 1 (3.7 \%) C, 7 (25.9\%) CRF07_BC, 17 (63.0 \%) CRF01_AE and 1 (3.7 \%) new recombinant were identified based on vif-env sequences (Table 2).

\section{HIV-1 group M subtype characterization in Suzhou and Suqian}

To determine genotype of each sample, the subtyping results of four fragments were taken into account together (Table 2). To assure the accuracy, only the samples with one available pol or vif-env sequence were included. According to this standard, four samples (11jssqHS005, 11jssq012, 11jssqHS008 and 12jsszIDU010) that lack both $p o l$ and vif-env sequences were excluded in the statistics. Among the remaining 34 samples, 7 (20.6 \%) CRF07_BC, 3 (8.8\%) CRF08_BC, 19 (55.9 \%) CRF01_AE, and 5 (14.7 \%) inter-subtype recombinants were found in two cities Suzhou and Suqian (Fig. 1b). No pure B, $\mathrm{B}^{\prime}$ and $\mathrm{C}$ subtypes were identified. Five recombinants contain one $B / C$, three $C R F 01 / B$ and one $\mathrm{CRF} 01 / \mathrm{B} / \mathrm{C}$ recombinants (Table 2). Because of having different recombination breakpoints, they represent five unique recombinant forms (URFs). These results indicated that multiple HIV-1 subtypes, including CRF01_AE, CRF07_BC, CRF08_BC and some URFs were circulating in Jiangsu, and the predominant HIV-1 genotype was CRF01_AE, followed by CRF07_BC and CRF08_BC (Fig. 1b).

In Suqian, 6 (54.5\%) CRF01_AE, 1 (9.1 \%) CRF07_BC, 2 (18.2\%) CRF08_BC, and 2 (18.2\%) URFs were identified (Fig. 1a). In Suzhou, 13 (56.5\%) CRF01_AE, 7 (26.1 \%) CRF07_BC, 1 (4.3\%) CRF08_BC, and $3(13.0 \%)$ URFs were identified (Fig. 1a). Although there is a long geographical distance between Suqian and Suzhou, there was no obvious difference in HIV-1 group $M$ subtype distribution between the two cities (Fig. 1). In particular, CRF01_AE appeared to be the most predominant HIV-1 strains circulating in both cities (Fig. 1a), consistent with the increasing trend of CRF01_AE prevalence in China (Zhang et al. 2014). 
Table 2 Subtype characterizations of four genomic fragments from HIV-infected population in Suzhou and Suqian, Jiangsu

\begin{tabular}{|c|c|c|c|c|c|}
\hline Specimen & $p 17$ & pol & vif-env & $c 2 v 3$ & Total $^{\mathrm{a}}$ \\
\hline 11jssq001 & C & CRF08_BC & NA & C & CRF08_BC \\
\hline 11jssqHS002 & CRF01_AE & CRF01_AE & CRF01_AE & NA & CRF01_AE \\
\hline 11jssqHS013 & CRF01_AE & CRF01_AE & NA & CRF01_AE & CRF01_AE \\
\hline 11jssqIDU011 & NA & NA & CRF07_BC & $B / C$ & $\mathrm{~B} / \mathrm{C}$ \\
\hline 11jssqMSM003 & CRF01_AE & CRF01_AE & CRF01_AE & CRF01_AE & CRF01_AE \\
\hline 11jssqMSM004 & CRF01_AE & CRF01_AE & CRF01_AE & CRF01_AE & CRF01_AE \\
\hline 11jssqMSM006 & $C$ & CRF08_BC & NA & NA & CRF08_BC \\
\hline 11jssqMSM007 & CRF01_AE & CRF01_AE & CRF01_AE & CRF01_AE & CRF01_AE \\
\hline 11jssqMSM009 & CRF01_AE & CRF01_AE & CRF01_AE & CRF01_AE & CRF01_AE \\
\hline 11jssqMSM010 & $C$ & CRF07_BC & CRF07_BC & C & CRF07_BC \\
\hline 11jssqMSM014 & NA & CRF01_AE/B & NA & CRF01_AE & CRF01_AE/B \\
\hline 12jssz001 & $C$ & CRF07_BC & CRF07_BC & C & CRF07_BC \\
\hline 12jsszHSO02 & CRF01_AE & CRF01_AE & CRF01_AE & CRF01_AE & CRF01_AE \\
\hline 12jsszHSO04 & CRF01_AE & CRF01_AE & CRF01_AE & CRF01_AE & CRF01_AE \\
\hline 12jsszHSO05 & $C$ & CRF07_BC & NA & C & CRF07_BC \\
\hline 12jsszHS008 & CRF01_AE & CRF01_AE & CRF01_AE & NA & CRF01_AE \\
\hline 12jsszHSO11 & CRF01_AE & NA & CRF01_AE & CRF01_AE & CRF01_AE \\
\hline 12jsszHSO12 & CRF01_AE & CRF01_AE & CRF01_AE & CRF01_AE & CRF01_AE \\
\hline 12jsszHSO13 & C & $B / C$ & CRF07_BC & CRF01_AE & CRF01_AE/B/C \\
\hline 12jsszHSO16 & $C$ & CRF07_BC & CRF07_BC & $C$ & CRF07_BC \\
\hline 12jsszHSO18 & CRF01_AE & CRF01_AE & CRF01_AE & CRF01_AE & CRF01_AE \\
\hline 12jsszHSO19 & CRF01_AE & CRF01_AE & CRF01_AE & CRF01_AE & CRF01_AE \\
\hline 12jsszHSO20 & CRF01_AE & CRF01_AE & CRF01_AE & CRF01_AE & CRF01_AE \\
\hline 12jsszHS021 & B & B & B & CRF01_AE & CRF01_AE/B \\
\hline 12jsszHSO22 & C & CRF08_BC & C & C & CRF08_BC \\
\hline 12jsszHSO23 & CRF01_AE & CRF01_AE & CRF01_AE/B & CRF01_AE & CRF01_AE/B \\
\hline 12jsszHSO24 & C & CRF07_BC & CRF07_BC & C & CRF07_BC \\
\hline 12jsszMSM003 & CRF01_AE & CRF01_AE & CRF01_AE & CRF01_AE & CRF01_AE \\
\hline 12jsszMSM006 & $C$ & CRF07_BC & CRF07_BC & C & CRF07_BC \\
\hline 12jsszMSM009 & CRF01_AE & CRF01_AE & NA & CRF01_AE & CRF01_AE \\
\hline 12jsszMSM014 & $C$ & CRF07_BC & NA & C & CRF07_BC \\
\hline 12jsszMSM015 & CRF01_AE & CRF01_AE & CRF01_AE & CRF01_AE & CRF01_AE \\
\hline 13jsszIDU017 & CRF01_AE & CRF01_AE & CRF01_AE & CRF01_AE & CRF01_AE \\
\hline 13jsszMSM007 & CRF01_AE & CRF01_AE & CRF01_AE & CRF01_AE & CRF01_AE \\
\hline Total & 32 & 32 & 27 & 31 & 34 \\
\hline
\end{tabular}

If no sequences are available on pol and vif-env, the strains were not taken into account in the statistics

NA not available

a CRF07_BC and CRF08_BC originated by insertion of several short segments of subtype B into the backbone of subtype C. Both CRFs_BC have genomic segments of subtype $C$ origin in 117 and $C 2$ V3 region. Therefore, it is unable to distinguish CRF07 BC/CRF08 BC from subtype $C$ in the phylogenetic trees of $p 17$ and $C 2$ V3. Therefore, the genomic segments (i.e. pol and vif-env) including recombination breakpoints are used as major determinants for identification of CRF07_BC or CRF08_ $\mathrm{BC}$

In China, CRF01_AE was the most predominant strains circulating in the sexual transmission group (including heterosexuals and homosexuals), and CRF07 BC and CRF08_BC were major circulating among injection drug users (IDUs) (Yang et al. 2002; Su et al. 2000; Piyasirisilp et al. 2000). We investigated the potential association between HIV-1 genotypes and the risk behaviors. In the heterosexuals, the most predominant strains were CRF01_AE (58.8 \%), followed by CRF07_BC (23.5\%). Similarly, in the homosexuals, the most predominant stains were also CRF01_AE (61.5\%), followed by CRF07_BC (23.1\%). In addition, among the only two IDUs, one was CRF01_AE and another was B/C recombinant. The emergences of relatively high proportions of CRF07_BC in the sexual transmission group and of CRF01_AE among IDUs might suggest an increasing 


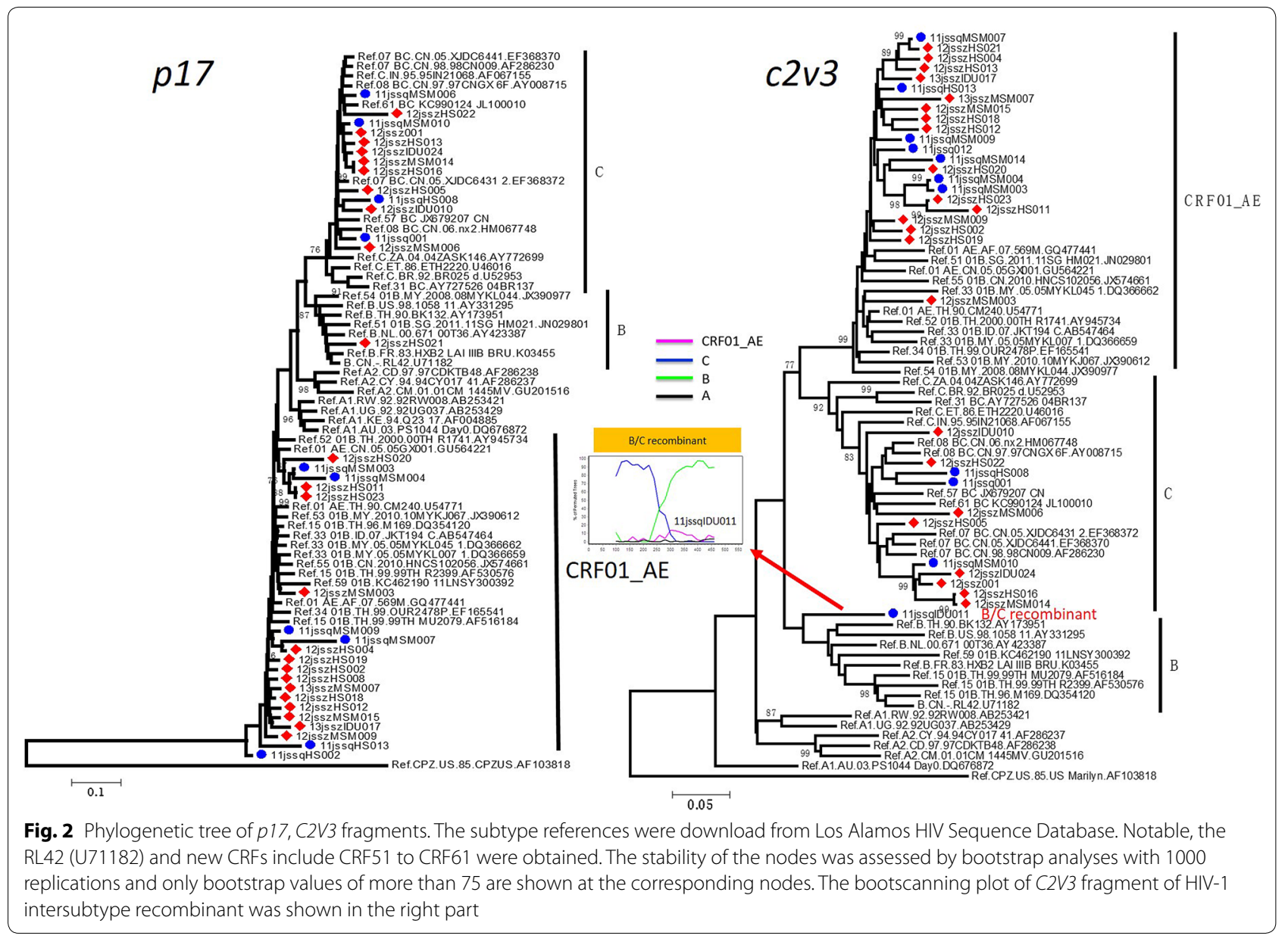

trend of HIV-1 transmission between different risk groups.

\section{Comparison of HIV-1 group M subtype distributions between Jiangsu and the surrounding provinces}

We further compared the HIV-1 group M subtype distribution between Jiangsu and the surrounding provinces. Because of no data available from Zhejiang, only the data from Shanghai, Shandong, and Anhui were covered. As the subtype results in previous studies were based on the pol sequences, comparison was performed only based on the results from pol sequences (Fig. 4). The comparison showed that Jiangsu had similar HIV-1 group M subtype distribution to the surrounding provinces. CRF01_AE was the most predominant strains in all four provinces with prevalence of $42.9-59.4 \%$. The second most common subtype was different in the four provinces with CRF07_BC in both Jiangsu and Anhui and subtype B in both Shanghai and Shandong. HIV-1 inter-subtype recombinants were identified in all four provinces with proportions of $4.2-10.6 \%$, and no or less pure subtype $\mathrm{C}$ strain was found there (Fig. 4). Because only pol region was used in HIV-1 genotyping, the actual numbers of HIV-1 inter-subtype recombinants in the four provinces (e.g., east China) might be greater than reported data. In contrast, the numbers of subtypes $B$ and $C$ might be lower than reported data. Most recombinants in these regions were CRF01_AE-associated recombinants, different from the observation in southwestern China, where $\mathrm{B} / \mathrm{C}$ recombinants were the most common recombinant forms (Pang et al. 2012).

\section{Discussion}

IDU and former commercial plasma donation were the two major modes of HIV transmission in China during 1985 to 2005, accounting for 44.2 and $29.6 \%$ of total infections, respectively (Ministry of Health of the People's Republic of China, Joint United Nations Programme on HIV/AIDS, World Health Organization 2012; Yan et al. 2006; Zhang et al. 2004). In recent years, the number of new HIV-1 infections caused by sexual contacts, including heterosexual and homosexual contacts, had increased rapidly and sexual transmission had become the primary mode of HIV infection in China (Ministry 


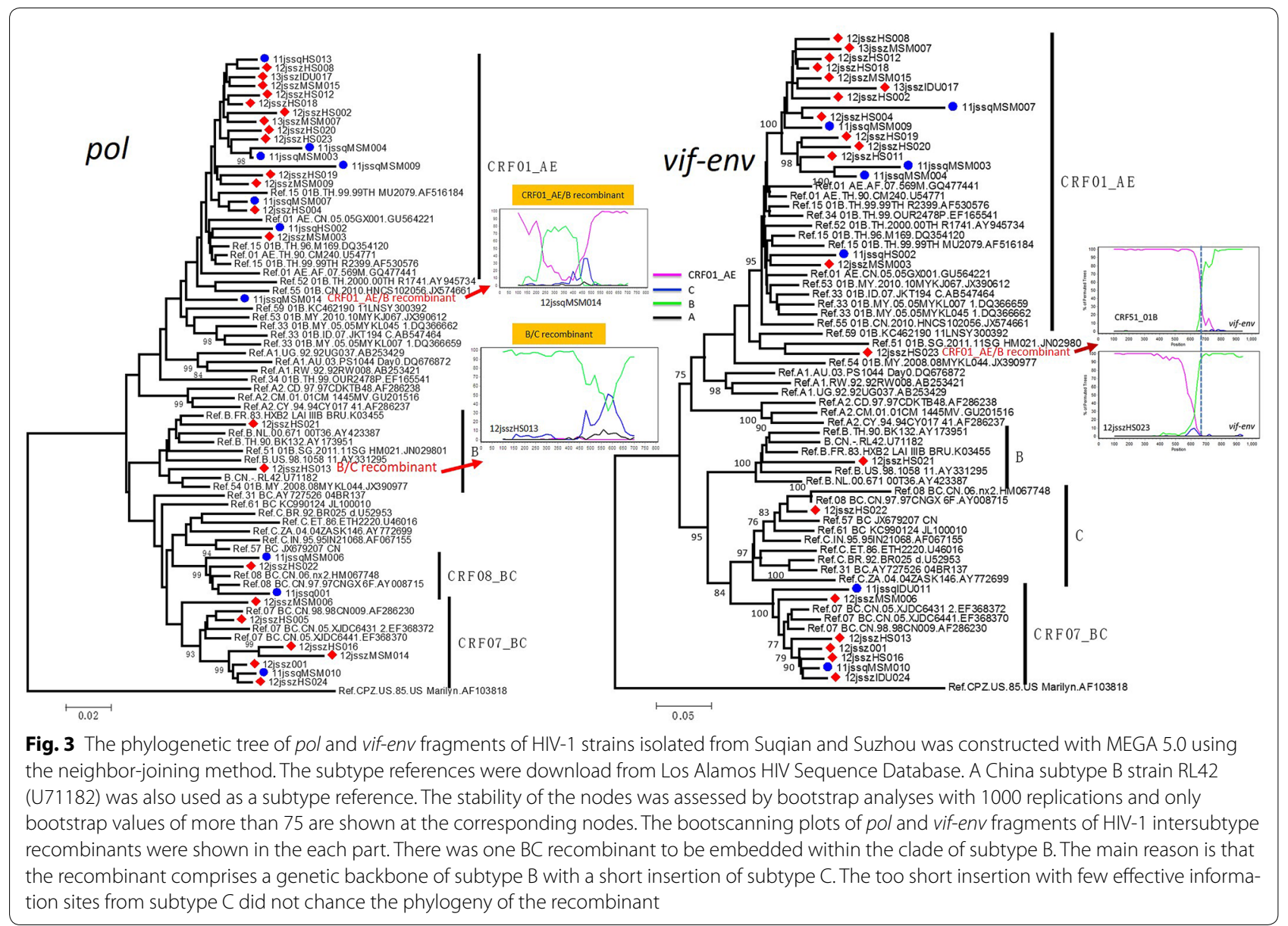

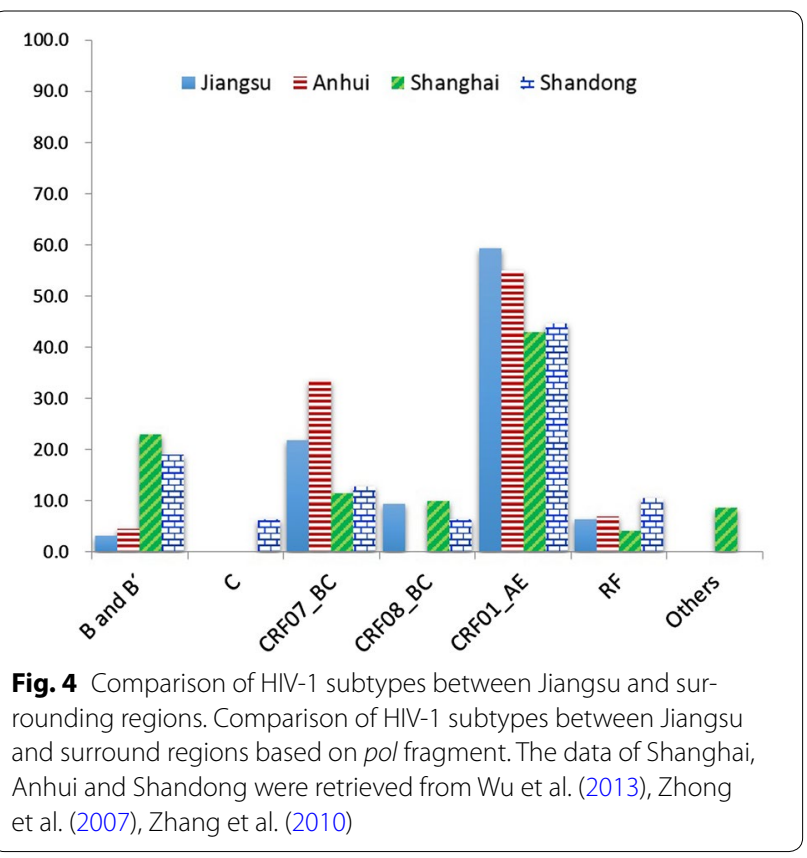

of Health of the People's Republic of China, Joint United Nations Programme on HIV/AIDS, World Health Organization 2012; Guo et al. 2009a; Wu et al. 2013; Ye et al. 2014; An et al. 2012; Xu et al. 2013). Among the 48,000 new infections in China in 2011, approximately $81.6 \%$ was associated with sexual exposure (Ministry of Health of the People's Republic of China, Joint United Nations Programme on HIV/AIDS, World Health Organization 2012). The similar trend could be also observed in Jiangsu. The investigation conducted in 2011 showed that $87.26 \%$ of PLHIV in Jiangsu acquired HIV-1 infection via sexual contact, including $46.6 \%$ heterosexual and $40.64 \%$ homosexual contacts (Control JPCfDPA 2012). Our results showed that $81.6 \%$ (heterosexuals: $50.0 \%$ and homosexual: $31.6 \%$ ) of HIV infected cases in Suzhou and Suqian acquired infections through sexual contacts, very close to the data of whole province. These imply that the prevention and control of HIV-1 should focus on sexual transmission group in Jiangsu. One difference between Suzhou and Suqian was that more HIV-infected individuals $(62.5 \%)$ in Suzhou have higher education level than 
in Suqian $(28.6 \%)$, which might be attributed to higher economic development and cultural education levels in Suzhou than in Suqian.

Jiangsu is one of the most developed areas in China (Fig. 1a), and serves as a crucial transportation hub linking Shanghai and other regions of China. Therefore, there are a large number of migrant populations from some undeveloped areas to work and live in Jiangsu or go through Jiangsu to Shanghai or Zhenjiang, another two most developed provinces in China, which may directly or indirectly facilitate the spread of and co-circulation of various human viruses or subtypes of certain virus in this area. Previous report showed that about $72.0 \%$ of HIV-positive persons in Jiangsu were living in Nanjing, Suzhou, Wuxi, Changzhou and Xuzhou cities (Control JPCfDPA 2012), all which are located in the important lines (including railway, high-speed railway and high-speed way)crossing Jiangsu. These may support a potential association between a large scale of population mobility and HIV transmission.

Previous results based on gag and $\mathrm{C} 2 \mathrm{V3}$ fragments showed that seven HIV-1 group $M$ subtypes, including B, C, CRF01_AE, CRF02_AG, CRF07_BC, CRF08_BC, and $A 1$ were circulating in Jiangsu (Yang et al. 2009). The co-circulation of multiple HIV-1 group M subtypes will increase the chance to generate new HIV-1 recombinant (Guo et al. 2014). In this study, we detected five (14.7\%) recombinants from 34 samples collected in Suzhou and Suqian. Apart from these recombinants, we also detected 19 (55.9 \%) CRF01_AE, 7 (20.6 \%) CRF07_BC, 3 (8.8 \%) CRF08_BC, but no pure subtype $B$ and $C$, showing an obviously different genotype distribution of HIV-1 group $\mathrm{M}$ with previous ones (Li et al. 2013). These possibly suggest a new molecular epidemiological trend of HIV-1 in Jiangsu.

HIV-1 genotype distribution in Jiangsu (including Suzhou and Suqian) was similar to those in the surrounding provinces (e.g., Shanghai, Anhui, and Shandong) (Wu et al. 2013; Zhong et al. 2007; Zhang et al. 2010). CRF01_AE appeared to be the most predominant HIV-1 genotypes in the four provinces (Fig. 4). Among five recombinants identified in Jiangsu, four (80\%) were CRF01_AE-involoved recombinants, including three CRF01/B and one CRF01/B/C recombinants, consistent with the observation in the three surrounding provinces, where $68.8 \%(11 / 16)$ of recombinants were CRF01_AE related (Wu et al. 2013; Zhong et al. 2007; Zhang et al. 2010). High proportion of CRF01_ AE-involved recombinants might be the result of high prevalence of CRF01_AE in the East China. In addition, only one B/C recombinant was identified in Suzhou and Suqian. Interestingly, no pure HIV-1 group M subtypes
$\mathrm{B}$ and $\mathrm{C}$ strains were found. It implies that there may be two reasons for the origin of these subtype $B$ and $\mathrm{C}$-involved recombinants. First, the $\mathrm{B}$ and $\mathrm{C}$ fragments of these recombinants might come from CRF07_BC or CRF08_BC. Second, these recombinants might originate in other regions where $B$ and $C$ subtypes were circulating and be introduced into Suzhou and Suqian. Furthermore, no finding of pure HIV-1 group M subtype B and $\mathrm{C}$ strains also implies that fewer $\mathrm{B} / \mathrm{C}$ recombinants will be generated in Jiangsu (at least in Suzhou and Suqian) in future. On the other hand, CRF01_AE and CRF07_BC were the top two predominant HIV-1 group $M$ subtypes in Jiangsu and Anhui (Fig. 4), which implies that more CRF01/CRF07 recombinants will be generated in the two provinces in future. In fact, two CRF01/CRF07 recombinants had been identified from one IDUs in Jiangsu and three MSM in Anhui previously (Wu et al. 2013; Guo et al. 2009b). Therefore, to effectively monitoring the changes of HIV-1 diversity in this area, a series of large-scale molecular epidemiological investigation are needed.

\section{Conclusion}

In this study, by characterizing the subtype distribution of HIV-1 group M in Suzhou and Suqian, we found that CRF01_AE was the most predominant HIV-1 group $M$ subtypes in Jiangsu, and less and/or no pure subtype $\mathrm{B}$ and $\mathrm{C}$ was currently circulating here. We predicted that more CRF01/CRF07 recombinants, but fewer B/C recombinants will be generated in Jiangsu in future. Additional, we also suggest that the actual number of HIV-1 inter-subtype recombinants is larger and the genetic diversity of HIV-1 group $M$ in Jiangsu is more complex than described here. Therefore, a molecular epidemiological investigation based on all high-risk groups in whole Jiangsu province is needed for providing detailed information for the prevention and control of HIV-1 in Jiangsu and even whole east China.

\section{Authors' contributions \\ Conceived and designed the experiments: HD. Performed the experiments: $\mathrm{CQ}, \mathrm{PZ}, \mathrm{FH}, \mathrm{WZ}$. Analyzed the data: CHQ, PZ, FH. Contributed reagents/materi- als/analysis tools: AG, PF, XZ. Wrote the paper: FH, HD. All authors read and approved the final manuscript.}

\section{Author details}

${ }^{1}$ Department of Clinical Laboratory, The Second Affiliated Hospital of Soochow University, 1055 Sanxiang Road, Suzhou 215004, Jiangsu, China.

${ }^{2}$ Department of Clinical Laboratory, Suqian Center of Disease Control and Prevention, Suqian, Jiangsu, China. ${ }^{3}$ Institute of Life Sciences, Jiangsu University, Zhenjiang, Jiangsu, China.

\section{Acknowledgements}

This study was supported by the startup funds from the Second Affiliated Hospital of Soochow University (No. SDFEYGJ1301).

\section{Competing interest}

The authors declare that they have no competing interests. 


\section{Ethics statement}

This study was done according to the Helsinki II Declaration and was approved by the medical ethics committee of Suqian Center of Disease Control and Prevention (CDC) and the Second Affiliated Hospital of Soochow University. Written informed consent was obtained from the patient for publication of this case report and any accompanying images.

Received: 27 January 2016 Accepted: 20 May 2016

Published online: 24 June 2016

\section{References}

An M, Han X, Xu J et al (2012) Reconstituting the epidemic history of HIV strain CRF01_AE among men who have sex with men (MSM) in Liaoning, northeastern China: implications for the expanding epidemic among MSM in China. JVirol 86:12402-12406

Control JPCFDPA, (2012) Analysis of AIDS epidemic in Jiangsu Province, 2011. Jiangsu J Prev Med 23:34-35

Guo H, Wei JF, Yang H et al (2009a) Rapidly increasing prevalence of HIV and syphilis and HIV-1 subtype characterization among men who have sex with men in Jiangsu, China. Sex Transm Dis 36:120-125

Guo HX, Guo DM, Wei JF et al (2009b) First detection of a novel HIV type 1 CRF01_AE/07_BC recombinant among an epidemiologically linked cohort of IDUs in Jiangsu, China. AIDS Res Human Retroviruses 25:463-467

Guo H, Hu H, Zhou Y et al (2014) A novel HIV-1 CRF01_AE/B recombinant among men who have sex with men in Jiangsu Province, China. AIDS Res Hum Retroviruses 30:706-710

Li L, Sun G, Liang S, Li J, Li T et al (2013) Different distribution of HIV-1 subtype and drug resistance were found among treatment naive individuals in Henan, Guangxi, and Yunnan province of China. PLoS ONE 8:e75777

Liu W, Chen J, Rodolph M et al (2006) HIV prevalence among injection drug users in rural Guangxi China. Addiction 101:1493-1498

Liu S, Xing H, He X et al (2008) Dynamic analysis of genetic diversity of gag and env regions of HIV-1 CRF07_BC recombinant in intravenous drug users in Xinjiang Uvghur Autonomous Region, China. Arch Virol 153:1233-1240

Lole KS, Bollinger RC, Paranjape RS et al (1999) Full-length human immunodeficiency virus type 1 genomes from subtype C-infected seroconverters in India, with evidence of intersubtype recombination. J Virol 73:152-160

Meng X, Wang L, Chan S et al (2011) Estimation and projection of the HIV epidemic trend among the migrant population in China. Biomed Environ Sci 24:343-348

Ministry of Health of the People's Republic of China, Joint United Nations Programme on HIV/AIDS, World Health Organization (2012) 2011 Estimates for the HIV/AIDS Epidemic in China. Chin J AIDS STD 18:1-5

$\mathrm{Ng}$ OT, Eyzaguirre LM, Carr JK et al (2012) Identification of new CRF51_01B in Singapore using full genome analysis of three HIV type 1 isolates. AIDS Res Hum Retroviruses 28:527-530

Pang W, Zhang CY, Duo L et al (2012) Extensive and complex HIV-1 recombination between B', C and CRF01_AE among IDUs in south-east Asia. Aids 26:1121-1129
Piyasirisilp S, McCutchan FE, Carr JK et al (2000) A recent outbreak of human immunodeficiency virus type 1 infection in southern China was initiated by two highly homogeneous, geographically separated strains, circulating recombinant form $\mathrm{AE}$ and a novel $\mathrm{BC}$ recombinant. J Virol 74:11286-11295

Su L, Graf M, Zhang Y et al (2000) Characterization of a virtually full-length human immunodeficiency virus type 1 genome of a prevalent intersubtype (C/B') recombinant strain in China. J Virol 74:11367-11376

Sun XG, Xing H, Li ZJ et al (2011) Distribution of HIV-1 subtype and its relationship with HIV/AIDS epidemic in Guizhou province]. Zhonghua Liu Xing Bing Xue Za Zhi 32:689-692

Tamura K, Peterson D, Peterson N et al (2011) MEGA5: molecular evolutionary genetics analysis using maximum likelihood, evolutionary distance, and maximum parsimony methods. Mol Biol Evol 28:2731-2739

Wu JJ, Meng ZF, Xu JQ et al (2013) New emerging recombinant HIV-1 strains and close transmission linkage of HIV-1 strains in the Chinese MSM population indicate a new epidemic risk. PLoS ONE 8:e54322

Xu J, An M, Han X et al (2013) Prospective cohort study of HIV incidence and molecular characteristics of HIV among men who have sex with men (MSM) in Yunnan Province, China. BMC Infect Dis 13:3

Yan J, Wang Y, Li J et al (2006) Subtype and sequence analysis of the ENV gene of HIV-1 drug users in Guangdong Pearl River delta.]. Zhonghua Shi Yan He Lin Chuang Bing Du Xue Za Zhi 20:223-225

Yang R, Xia X, Kusagawa S et al (2002) On-going generation of multiple forms of HIV-1 intersubtype recombinants in the Yunnan Province of China. AIDS 16:1401-1407

Yang HT, Qiu T, Huan XP et al (2009) Molecular epidemiology of the human immunodeficiency virus-1 isolated from patients confirmed lately in Jiangsu province. Acta Univ Med Nanjing Nat Sci 29:976

Ye JR, Zang WC, Su XL et al (2014) Molecular epidemiological characteristics of HIV-1 strains isolated from newly diagnosed MSM subjects (2006-2010) in Beijing, China. Bing Du Xue Bao 30:138-142

Zhang C, Yang R, Xia X et al (2002) High prevalence of HIV-1 and hepatitis C virus coinfection among injection drug users in the southeastern region of Yunnan, China. J Acquir Immune Defic Syndr 29:191-196

Zhang L, Chen Z, Cao Y et al (2004) Molecular characterization of human immunodeficiency virus type 1 and hepatitis C virus in paid blood donors and injection drug users in china. JVirol 78:13591-13599

Zhang J, Kang D, Fu J et al (2010) Surveillance of transmitted HIV type 1 drug resistance in newly diagnosed HIV type 1-infected patients in Shandong Province, China. AIDS Res Hum Retroviruses 26:99-103

Zhang L, Chow EP, Jing J, Zhuang X et al (2013) HIV prevalence in China: integration of surveillance data and a systematic review. Lancet Infect Dis 13:955-963

Zhang W, Han X, An M et al (2014) Identification and characterization of a novel HIV-1 circulating recombinant form (CRF59_01B) identified among men-who-have-sex-with-men in China. PLoS ONE 9:e99693

Zhong P, Pan QC, Ning Z et al (2007) Genetic diversity and drug resistance of human immunodeficiency virus type $1(H I V-1)$ strains circulating in Shanghai. AIDS Res Hum Retroviruses 23:847-856

\section{Submit your manuscript to a SpringerOpen ${ }^{\circ}$ journal and benefit from:}

- Convenient online submission

- Rigorous peer review

- Immediate publication on acceptance

- Open access: articles freely available online

- High visibility within the field

- Retaining the copyright to your article

Submit your next manuscript at $\boldsymbol{\nabla}$ springeropen.com 